\title{
Mechanical behavior of low carbon steel subjected to strain path changes: experiments and modeling
}

\author{
W. Wen ${ }^{\mathrm{a}}$, M. Borodachenkova ${ }^{\mathrm{a}, *}$, C.N. Toméb, G. Vincze ${ }^{\mathrm{a}}$, E.F. Rauch ${ }^{\mathrm{c}}$, F. Barlat ${ }^{\mathrm{a}, \mathrm{d}}$, J.J. Grácio ${ }^{\mathrm{a},+}$ \\ ${ }^{a}$ Center for Mechanical Technology and Automation, Department of Mechanical Engineering, \\ University of Aveiro, 3810-193 Aveiro, Portugal \\ ${ }^{b}$ Materials Science and Technology Division - Los Alamos National Laboratory - Los Alamos, \\ NM 87545 USA \\ ${ }^{c}$ Science et Ingénierie des Matériaux et des Procédés - (CNRS UMR 5266) INPG-UJF, BP 46, \\ 38402 Saint Martin d'Hères cedex, France \\ ${ }^{d}$ Graduate Institute of Ferrous Technology (GIFT), Pohang University of Science and \\ Technology (POSTECH), 77 cheongam-ro, Nam-gu, Pohang, Gyeongbuk 790-784, Republic of \\ Korea. \\ * Corresponding author: mborodachenkova@ua.pt \\ Tel. +351234370 827, Fax. +351234370953 \\ + Paper dedicated to the memory of Professor José Joaquim de Almeida Grácio (1959-2014)
}

\begin{abstract}
The mechanical response of a low carbon steel under complex strain path changes is analyzed here in terms of dislocation storage and annihilation. The mechanical tests performed are cyclic shear and tensile loading followed by shear at different angles with respect to the tensile axis. The material behavior is captured by a dislocation-based hardening model, which is embedded in the Visco-Plastic Self-Consistent (VPSC) polycrystal framework taking into account the accumulation and annihilation of dislocations, as well as back-stress effects. A new and more sophisticated formulation of dislocation reversibility is proposed. The simulated flow stress responses are in good agreement with the experimental data. The effects of the dislocation-related mechanisms on the hardening response during strain path changes are discussed.
\end{abstract}

Keywords: Crystallographic dislocation model, Microstructures, Strain path change, 
Polycrystalline material.

\section{Introduction}

The strain path changes that take place during industrial forming processes of metallic systems have been studied by several authors during the last decade [1-7]. Investigations show that the strain path change can affect the evolution of dislocation-related microstructure and thus leads to evident changes in the yield stress and flow stress evolution [7-12]. When the shear on a slip system is reversed, the internal back-stress induced during the previous loading usually facilitates the activation of glide on the reverse direction, leading to the Bauschinger effect [13-19]. As consequence, the flow stress is significantly lower than for the monotonic stress-strain curve during several percents of strain after reloading. Moreover, after the strain path is changed, the dislocation structure generated during previous loading is gradually dissolved and replaced by the structure being created in the new strain path [1].

The modeling of the hardening behavior of polycrystals subjected to monotonic strain or complex strain path changes has been recently addressed by several authors [7,10,20-24]. Among the different models proposed, the continuum approach by Rauch et al. [9], called RGVB model from now on, explicitly accounts for the dislocation accumulation/annihilation under strain reversal.

Kitayama et al. reformulated the RGBV model for a crystallographic framework [25]. This improved RGBV model tracks the dislocation density evolution on each slip system in each grain for two populations, namely, forward and reversible dislocations. During monotonic loading the evolution of dislocation density for both populations is described 
using the Kocks-Mecking approach. If the shear on the slip system is reversed, only the reversible dislocations are allowed to glide on the opposite direction and have the potential to recombine. A reversibility parameter $P$ was introduced to measure the fraction of reversible dislocations generated as a function of strain. $(1-P)$ measures the fraction of non-reversible dislocations. This parameter is assumed to be equal for all slip systems in one grain and calculated as a function of accumulated debris density. It is set to be 1 at the beginning of deformation and decreases gradually to 0 with increasing accumulated strain. This model was embedded in the visco-plastic self-consistent (VPSC) framework, which allows the grain orientation and crystallography to be accounted for explicitly. The information about strain path changes does not need to be enforced empirically but is implicitly built in the model by keeping track of shear reversals in each slip system during the simulations. Kitayama et al. [25] applied this model to predict the response of a rolled low carbon (LC) steel sheet subjected to uniaxial tension followed by simple shear reloading along different directions, and forward-reverse simple shear tests. Note that the formulation does not account for the Bauschinger effect and that the proposed evolution of the reversibility parameter $P$ is such that about $50 \%$ accumulated strain $P$ is about 0.5 and at $80 \%$ accumulated strain the generation rate of reversible dislocations becomes negligible.

More recently, Wen et al. [26] successfully extended the crystallographic RGBV model to HCP materials by including a formulation for the back-stress acting on the slip systems, and by accounting for twinning contribution to deformation. The effect of the back-stress is introduced as a correction term to the CRSS of each system depending on 
the density of reversible dislocations stored in such system.

In the present work, the simulations for tension-shear tests on LC steel done by Kitayama et al. [25] are repeated using the new formulation of the Bauschinger effect to evaluate the flow stress response during the initial $1-2 \%$ strain after reloading. In addition, the crystallographic RGBV model is employed for simulating cyclic loading of this LC steel up to very large strain amplitudes. A new formulation of the reversibility parameter $P$ is proposed since the previous version reduces $P$ monotonically with the accumulated strain and (incorrectly) predicts increasingly larger hysteresis loops in the case of cyclic loading. In the new formulation, the parameter $P$ is calculated individually for each slip system $s$ instead of being equal for all systems in one grain. It is also assumed to vary according to the current states of dislocation configurations.

\section{Crystallographic RGBV model}

The crystallographic RGBV model allows tracking down the dislocation density evolution in each slip system inside each grain throughout the plastic deformation process. The dislocations accumulated during pre-strain may be annihilated progressively during strain path changes. This dislocation evolution model was proposed by Kitayama et al. [25] who implemented it in the VPSC polycrystal framework $[27,28]$. The model tracks down the direction of shear in each slip system of each grain and identifies shear strain reversals induced by changes of the strain path or by grain reorientation associated with large strain plastic deformation.

The relation between dislocations and the critical resolved shear stress (CRSS) $\tau_{d}^{s}$, given by a form of the Taylor law extended to describe the latent hardening associated 
with dislocation-dislocation interactions within various materials as discussed by [2936], is used in this work instead of the simpler approach by Kitayama et al. [25] for BCC steel:

$\tau_{d}^{s}=\tau_{0}^{s}+\mu b^{s} \sqrt{\alpha^{s s} \rho^{s}+\sum_{s \neq s^{\prime}} \alpha^{s s^{\prime}} \rho^{s^{\prime}}}$

here, $\rho^{s}$ is the dislocation density on system $s, \tau_{0}^{s}$ the initial CRSS, $\mu$ the shear modulus, $b^{s}$ the magnitude of the Burgers vector and $\alpha^{s s^{\prime}}$ the latent hardening matrix.

In the model of Kitayama et al., the dislocation density on each slip system is comprised of forest and reversible dislocations [25]. The latter can recombine when shear on a slip system is reversed, which is in fact a mechanism of dislocation annihilation. Reversible dislocations are also responsible for a back-stress contribution $\Delta \tau_{B}^{s}$ to the CRSS on the slip system $s$, such that:

$\tau^{s}=\tau_{d}^{s}+\Delta \tau_{B}^{s}$

as discussed in Section 2.2. In order to introduce the reversible dislocations in the model, each slip system $s$ is split into $s^{+}$and $s^{-}$, which correspond to the activation of slip in the arbitrarily defined positive and negative direction of the Burgers vector. Note that when the system shears in one direction $\left(d \gamma^{s+(\text { or }-)}>0\right)$, the opposite system is inactive $\left(d \gamma^{s-(\text { or }+)}=0\right)$. The dislocation density on system $\mathrm{s}$ is decomposed as:

$\rho^{s}=\rho_{\text {for }}^{s}+\rho_{\text {rev }}^{s+}+\rho_{\text {rev }}^{s-}$

where $\rho^{s}$ denotes the total dislocation density on slip system $s, \rho_{\text {for }}^{s}$ is the forest or non-reversible dislocation density, $\rho_{\text {rev }}^{s+}$ and $\rho_{\text {rev }}^{s-}$ represent the reversible dislocation density on $s^{+}$and $s^{-}$, respectively. The total dislocation density in one grain is given by: 
$\rho=\sum_{s} \rho^{s}=\sum_{s}\left(\rho_{\text {for }}^{s}+\rho_{\text {rev }}^{s+}+\rho_{\text {rev }}^{s-}\right)$

The crystallographic RGBV model relies on the Kocks-Mecking concept [37] of a storage term and a recovery term for the evolution of each component, with the added assumption that a fraction $P^{s}$ of the stored dislocations can be reversed. When the slip system contributes 'positive' shear $\left(d \gamma^{s+}>0 ; d \gamma^{s-}=0\right)$, the increment of $\rho_{\text {for }}^{s}$ and $\rho_{\text {rev }}^{s}$ are given by:

$$
\begin{aligned}
& d \rho_{\text {for }}^{s}=\left(1-P^{s}\right) \frac{d \gamma^{s+}}{b \Lambda}-f \rho_{\text {for }}^{s} d \Gamma \\
& d \rho_{\text {rev }}^{s+}=P^{s} \frac{d \gamma^{s+}}{b \Lambda}-f \rho_{\text {rev }}^{s+} d \Gamma \\
& d \rho_{\text {rev }}^{s-}=-\frac{1}{b \Lambda}\left(\frac{\rho_{\text {rev }}^{s-}}{\rho_{0}^{s}}\right)^{m} d \gamma^{s+}
\end{aligned}
$$

here $\Lambda$ is a dislocation mean free path defined by:

$\frac{1}{\Lambda}=\frac{\sqrt{\rho}}{K}+\frac{1}{D}$

where $\rho$ is the total dislocation density in one grain given by Eq. (4). $K$ is the number of forest dislocations that a moving segment is able to cross before being immobilized by the obstacles and $D$ is the average grain size. In Eq. (5) $d \Gamma$ is used in the thermal recovery term instead of $d \gamma^{s+}$ because the concurrent slip on any of the slip systems can facilitate thermally activated recombination on a particular slip system [9]. In the case of monotonic loading, the sum of the first two terms in Eq. (5) over all systems gives the classic Kocks-Mecking evolution law for the total dislocation density [25,37]. The reversibility fraction $P^{\mathrm{s}}$ is a function of accumulated dislocation density and, different from Kitayama et al. [25] and Wen et al. [26], it is assumed here to depend on the specific activity of each system. In the initial deformation stages all dislocations generated are assumed to be reversible $\left(P^{s}=1\right)$ and, as dislocations accumulate, the 
reversibility decreases towards $P^{s}=0$. The evolution of the reversibility fraction with dislocation density is discussed in Section 2.3.

The last term in Eq. (5) describes the recombination of reversible dislocations $\rho_{\text {rev }}^{s-}$ created on system $s$ during the previous deformation history, in a manner inspired by the 'areal glide' with 'percolation/debris-accumulation' model of Kocks [13]. $\rho_{0}^{s}$ is the total density in the system when reversal starts. These dislocations follow a different evolution law than the 'forward' generated dislocations $\rho_{\text {rev }}^{s+}$ : they only annihilate among themselves when $d \gamma^{s+}>0$. This term is meant to capture the fact that a moving dislocation tends to leave a certain amount of debris around obstacles ('hard areas' in [13]). When shear reversal occurs the dislocation will sweep back the same area and may fully or partially recombine with the previously generated debris [9]. While the parameter $m$ was equal to 1 in the formulation of Kitayama et al. [25], Wen et al. [26] argue that $m$ should be lower than 1 in order to provide a faster rate of recombination for the reversible dislocations during the reversal process ( $m=0.5$ in the present work).

The activation of slip systems on positive and negative directions are equivalent. Thus, the expressions for dislocation density evolution when $s^{-}$is activated $\left(d \gamma^{s-}>0, d \gamma^{s+}=0\right)$ are:

$$
\begin{aligned}
& d \rho_{\text {for }}^{s}=\left(1-P^{s}\right) \frac{d \gamma^{s-}}{b \Lambda}-f \rho_{\text {for }}^{s} d \Gamma \\
& d \rho_{\text {rev }}^{s+}=-\frac{1}{b \Lambda}\left(\frac{\rho_{r e v}^{s+}}{\rho_{0}^{s}}\right)^{m} d \gamma^{s-} \\
& d \rho_{\text {rev }}^{s-}=P^{s} \frac{d \gamma^{s-}}{b \Lambda}-f \rho_{\text {rev }}^{s-} d \Gamma
\end{aligned}
$$

Eqs. (5) and (7) describe the dislocation 'storage' and 'unstorage' processes. For example, when the shear is activated on the positive direction $\left(d \gamma^{s+}>0, d \gamma^{s-}=0\right)$, the forward 
dislocation density $\rho_{\text {for }}^{s}$ and the reversible dislocation density along the same direction $\left(\rho_{\text {rev }}^{s+}\right)$ will be accumulated according to Eq. (5). The density stored during previous loading in the opposite direction $\left(\rho_{\text {rev }}^{s-}\right)$ will be gradually annealed, or remain null otherwise. When shear is reversed $\left(d \gamma^{s-}>0, d \gamma^{s+}=0\right), \rho_{\text {rev }}^{s-}$ and $\rho_{\text {for }}^{s}$ will start to accumulate, and the previously stored $\rho_{\text {rev }}^{s+}$ will be gradually annealed until its value reaches 0 or the shear rate in the system is reversed again. The evolution of the dislocation density for all three populations is tracked in this way throughout the entire simulation. Note that this model is embedded in the crystallographic VPSC framework which is able to deal with the slip directionality at the grain level depending on grain orientation, loading conditions, etc. Therefore, the sign of shear rate and the occurrence of slip reversal are determined explicitly during the simulations and no external input is required to "inform" the model when and in which direction the strain path is changed, as was the case in previous works $[9,11]$.

\section{$2.1 \quad$ Back-stress effects}

According to Eq. (2), two mechanisms determine the CRSS required to activate a system: the first is the evolution of dislocation density via Eq. (1) while the second is associated with the back-stress acting on dislocations, an effect not accounted for by Kitayama et al. [25]. During the forward loading, a dislocation substructure is generated in the material, known to be associated with polarized back-stresses [38,39]. In particular, a heterogeneous distribution of dislocations leads to long-range intragranular stresses [3941]. Consequently, it is reasonable to associate the residual stresses to the current state of the substructure of trapped dislocations. Loosely tangled (pile-up-type) reversible 
dislocations will induce a large back-stress, and more tangled ones will have their field screened and provide a smaller back-stress. Upon strain path change, the trapped dislocations are progressively remobilized and recombine. In the process, the dislocation density decreases due to recombination and, as a consequence, so does the back-stress. The trend is particularly evident when the macroscopic strain is reversed, leading to the well-known Bauschinger effect [13].

Introducing an empirical back-stress term in the effective resolved shear stress has been applied in previous works $[17,19]$ to model the Bauschinger effect. Here, rather than attempting to evaluate the back-stress, we adopt the alternative empirical approach of defining a lower effective CRSS required for activating the opposite shear. When the shear on $s$ is reversed, a lower resolved shear is required to reverse the loosely tangled dislocations (i.e.: as in the case of a pile-up), which is equivalent to assigning a lower threshold stress to dislocation activation. In order to capture the lowering of the backstress associated with the progressive recombination of the dislocations upon reversal, we relate reversible dislocations with back-stress through an empirical power law, as follows:

$$
\begin{aligned}
& \Delta \tau_{B}^{s}=-\tau_{d}^{s} f_{B}^{s}\left(\frac{\rho_{r e v}^{s-}}{\rho^{s}}\right)^{q} \quad \text { if } d \gamma^{+}>0 \\
& \Delta \tau_{B}^{s}=-\tau_{d}^{s} f_{B}^{s}\left(\frac{\rho_{r e v}^{s+}}{\rho^{s}}\right)^{q} \quad \text { if } d \gamma^{-}>0
\end{aligned}
$$

Hence, the effective CRSS for system $s$ is expressed as:

$$
\tau^{s}=\tau_{d}^{s}+\Delta \tau_{B}^{s}=\tau_{d}^{s}\left[1-f_{B}^{s}\left(\frac{\rho_{r e v}^{s-(+)}}{\rho^{s}}\right)^{q}\right] \text { if } d \gamma^{+(-)}>0
$$

By construction, the back-stresses are directly related to the reversible dislocations 
density. Since according to Eqs. (5) and (7) the reversible dislocations recombine during shear reversals on the system, their effect on the effective CRSS decreases gradually and the back-stresses eventually vanish. $f_{B}^{s}\left(0<f_{B}^{s} \leq 1\right)$ in Eq. (8) is the scaling factor that weights the coupling between reversible dislocations and back-stresses. The parameter $q$ controls how fast the back-stress effect is nullified. It should be noted that the crystallographic RGBV model described above applies to the hardening evolution of slip systems. Twinning and its contribution to hardening was incorporated in the RGBV model by Wen et al [26] for studying tensile reloads in rolled Mg alloy.

\subsection{The reversibility parameter}

The formulation described above relies on a 'reversibility parameter' $P^{\mathrm{s}}$, which characterizes the fraction of dislocations generated in system $s$ that are reversible. Such fraction should, in general, decrease with monotonic deformation, as the dislocation densities and so the density of obstacles increase. The evolution of the reversibility fraction is empirically described by the sigmoidal function:

$$
P^{s}\left(D^{s}\right)=0.5-0.5 \tanh \left[3\left(\frac{\rho_{\max }+\rho_{\min }}{\rho_{\max }-\rho_{\min }}\right)\left(\frac{\sqrt{D^{s}}-\rho_{\text {infl }}}{\rho_{\text {infl }}}\right)\right]
$$

where $\sqrt{D^{s}}$ is a measure of the dislocation density representing an obstacle to dislocation reversal on system $s$. The characteristic densities $\rho_{\max }$ and $\rho_{\min }$ can be regarded as material properties which determine the value of $\sqrt{D^{s}}$ for which the parameter $P^{\mathrm{s}}$ goes from one to zero, respectively, and $\rho_{\mathrm{infl}}=\frac{\rho_{\max }+\rho_{\min }}{2}$ is the inflexion point of the sigmoidal. In the formulation of Kitayama et al. [25], the 'obstacle measure' parameter (here called $\sqrt{D^{s}}$ ) was assumed to increase monotonically with strain, and to 
be the same for every system in each grain, although different from grain to grain. The large strain cycling of LC steel studied here suggested a revision of its definition by assuming that $\sqrt{D^{s}}$ is different for each individual system and that it can decrease if the obstacles to dislocation glide on system $s$ decrease. As a consequence, it differs from the measure proposed in Kitayama et al. [25] and Wen et al. [26] while being equally successful at reproducing the results reported by the latter authors. This new measure of obstacle evolves incrementally with deformation and depends on the deformation history as:

$$
D^{s}=\int_{0}^{\varepsilon} \Delta D^{s}
$$

where

$$
\Delta D^{s}=\Delta \rho^{s} \sum_{s^{\prime}} \rho^{s^{\prime}}\left(1-\left|\bar{n}^{s} \cdot \bar{n}^{s^{\prime}}\right|\right)
$$

The evolution of obstacles acting on system $s$ depends on the system activity through $\Delta \rho^{s}$ and its coupling with existing dislocations through $\rho^{s^{\prime}}$. The strength of dislocation junctions exhibits a complex dependence on their relative orientations and the crystallography $[29,32,33,42-45]$. In the present model, we propose a simple form of coupling which is meant to favor reversibility of coplanar dislocations without getting into the details of dislocation junctions. The coupling between $s$ and $s^{\prime}$ is modulated by the angle between the glide planes via the factor $\left(1-\left|\bar{n}^{s} \cdot \bar{n}^{s^{\prime}}\right|\right)=1-\cos \left(\bar{n}^{s} \bar{n}^{s^{\prime}}\right)$. Such factor is zero if the systems are coplanar, equal to one if the glide planes intersect at $90^{\circ}$, and intermediate otherwise. This solution is simple enough and similar in structure to that used in $[25,26]$. This new formulation was implemented into the VPSC code and its 
performance is analyzed in the following sections.

\subsection{Brief description of the VPSC model}

The VPSC (visco-plastic self-consistent) model is described in detail by Lebensohn et al. [27] and Wang et al. [46]. For the purpose of this work it suffices to state that the VPSC framework is used as a platform for calculating the interaction between the effective medium (representing the polycrystal) and an ellipsoidal inclusion (representing the grain) hardening according to the constitutive law discussed above. In this work the aggregate is represented by a 1000 crystal orientations with weights chosen to reproduce the initial texture. When a loading path (strain history) for the polycrystal is simulated, VPSC predicts the macroscopic stress-strain response, the slip activity and dislocation evolution in each grain, and crystal reorientation (texture evolution). The plastic strain rate in each grain occurs via the combined contribution of the shear rates of all slip and twinning systems. The latter are related to the stress in the grain through the constitutive law:

$\dot{\varepsilon}_{i j}^{g}=\sum_{s} m_{i j}^{s} \dot{\gamma}^{s}=\dot{\gamma}_{0} \sum_{s} m_{i j}^{s}\left(\frac{m_{k l}^{s}: \sigma_{k l}^{g}}{\tau^{s}}\right)^{n}$

where $m_{i j}^{s}=\frac{1}{2}\left(n_{i}^{s} b_{j}^{s}+n_{j}^{s} b_{i}^{s}\right)$ is the symmetric Schmid tensor associated with slip system $s ; \bar{n}^{s}$ and $\bar{b}^{s}$ are the normal and burgers vector of the system; $\tau^{s}$ is the CRSS which is determined by the RGBV model (Eq. 9); $\dot{\varepsilon}_{i j}^{g}$ and $\sigma_{k l}^{g}$ are the deviatoric strain-rate and stress of the grain; $\dot{\gamma}_{0}$ is a normalization rate and $n$ the rate-sensitivity exponent.

\section{Results and discussion}


The simulations are performed within the RGVB-VPSC framework described in the previous section. Notice that the visco-plastic modeling framework does not include elasticity, and so is not able to capture the initial elastic effects associated with loading. In order to compare measured and predicted stress-strain response, the elastic part of the experimental data is excluded. The initial texture represented with 1000 grains is used as input to the VPSC model as in Kitayama et al. [25]. The intermediate grain-medium interaction $n^{\text {eff }}=10$ is chosen and the inverse strain-rate sensitivity $n$ is taken as 20 . The plastic deformation is assumed to be accommodated by $\{110\}\langle 111\rangle$ and $\{112\}\langle 111\rangle$ slip. The simulations are performed sequentially throughout the entire deformation process. The calculated deformation state at the end of one process is considered as the initial state of the following stage. The macroscopic boundary conditions for the uniaxial tension and simple shear tests are the same as in Kitayama et al. [25]. All the simpleshear tests are simulated by fully imposing the macroscopic velocity gradient $L_{i j}=\partial \dot{U}_{i} / \partial X_{j}$. For the uniaxial tension tests the velocity gradient component $L_{11}$ is enforced, together with the stress-free condition along axial directions 2 and 3 ( $\left.\Sigma_{22}=\Sigma_{33}=0\right)$. In this section, the experimental results of tension-shear and cyclic shear tests are used to evaluate the model. These two sets of experiments were performed on different grades of LC steels. The hardening parameters for both tests, which are listed in Table 1, are obtained by backfitting the experimental mechanical behavior.

\subsection{Tension preload followed by shear}

Fig. 1 presents the experimental [47] and predicted stress-strain response for tensile preloads of $\varepsilon_{11}=5 \%, 10 \%$ and $20 \%$ along the RD, each followed by shear reload at $45^{\circ}$, 
$90^{\circ}$ and $135^{\circ}$ with respect to the RD. The previous simulations of Kitayama et al. [25] were repeated here using the new formulation for the back-stress and reversibility of dislocations.

As reported by Rauch and Schmitt [47], the mechanical response depends strongly on the angle between the tensile and the shear directions. Uniaxial tension pre-strain produces the maximum resolved shear stress at about $45^{\circ}$ from the longitudinal axis. When reloading in simple shear at $45^{\circ}$ from the uniaxial axis, a large amount of dislocations continue gliding along the previous system and the occurrence of shear reversal is rare. For reloading at $90^{\circ}$, the highest flow stress response occurs: in this case, the slip systems activated during pre-loading (in tension) become latent and can act as obstacles for slip on the newly activated slip systems, resulting in an increase of the yield stress after the strain path change. For shear reloading at $135^{\circ}$ from the RD, the slip on many dislocation systems is reversed shear, resulting in a softening and Bauschinger effect.

Fig. 1 indicates that the mechanical responses in this set of experiments are captured by the RGBV-VPSC framework. The Bauschinger effect is achieved after shear reloading along $135^{\circ}$. The activation of the back-stress affects the relative activities for both families of slip systems as well. As shown in Fig. 2, the relative activities for simple shear reloading at $135^{\circ}$ deviate from those of the other reloading modes, an effect which was not captured in the previous work [25]. The pole figures for $10 \%$ tension pre-strain followed by $90^{\circ}$ shear reloading are presented in Fig. 3. These pole figures are similar to those in Kitayama et al. [25], indicating that texture evolution is rather insensitive to the 
new formulation of the reversibility parameter $P^{s}$ and the new back-stress law. Notice that the corresponding pole figure axes for the tension-shear and shear-shear tests in Figs 3 and 9 of Kitayama et al. [25] are mislabeled.

\subsection{Cyclic shear loading}

Cyclic forward-reverse simple shear tests have been carried out on the rolled LC steel with strain amplitudes: $\pm 10 \%, \pm 20 \%, \pm 30 \%$ and $\pm 40 \%$ shear strain $\left(\varepsilon_{12}=\gamma / 2\right)$ along the RD [48]. The measured flow stress responses, presented in Fig. 4, show that the mechanical response after one or two cycles reaches a saturation stage until failure occurs. As shown in Fig. 5, the predicted strain-stress responses are in good agreement with experimental observations: both hardening and saturation stages associated with cyclic tests are well captured by the crystallographic RGBV model. The strong Bauschinger effect after each shear reversal follows from the back-stress law (Eq. 8), but does not change the dislocation density in any meaningful way. The work-hardening stagnation, on the other hand, is a direct consequence of reversible dislocation annihilation. Fig. 6 presents the predicted flow stress behavior after 5 cycles for the strain amplitude of $\pm 20 \%$. It shows that the cyclic behavior is well described even for a large cumulated strain. The predicted pole figures for cyclic loading with $\pm 30 \%$ strain amplitude are presented in Fig. 7, and show that the texture also exhibits a cyclic behavior, remaining essentially the same at the end of each amplitude cycle. Notice that the texture evolution depends on the shear activity of the slip systems, which is mainly determined by the strain mode imposed. Some of these strain comes from the shear reversal mechanisms in the RGBV model. The latter affects the flow stress but has little 
effect on the predicted textures.

Fig. 8 shows that the evolution of forward and reversible dislocation densities is also periodic upon the attainment of saturation. In the simulation, such a behavior is due to the equilibrium between dislocation generation and annihilation. Fig. 9 schematically represents the evolution of the forward and reversible dislocation densities on one representative slip system during cyclic loading, obtained by imposing shear to a single crystal and disabling the update of grain orientation. As described in Section 2, the reversible dislocations on $s^{+}$and $s^{-}$experience the accumulation or annihilation processes depending on the sign of $\dot{\gamma}^{s}$. Therefore, after each reversal, $\rho_{\text {rev }}^{s+}$ and $\rho_{\text {rev }}^{s-}$ increase and decrease alternatively and achieve an equilibrium status at the saturation stage, which leads to the cyclic evolutions of dislocation density and flow stress. Notice that in the simulations the actual evolutions of $\rho_{\text {rev }}^{s+}$ and $\rho_{\text {rev }}^{s-}$ for each slip system may be affected by the update of grain orientation. However, a cyclic behavior in the total dislocation density evolution and strain-stress response can still be achieved in the aggregate as a whole.

The new formulation of the dislocation reversibility parameter $P^{s}$ also plays an important role in capturing the mechanical behavior under cyclic loading. In the formulation of Kitayama et al. [25], this parameter was defined as a function of debris density. Since the latter was only allowed to increase monotonically with deformation, the sigmoidal functional form of the reversibility parameter (given by Eq. 10) decreased monotonically from 1 to 0 , irrespective of whether the strain path exhibits reversal or not. As a consequence, when the accumulated strain is very large (i.e. cyclic loading test), 
the fraction of $\rho_{\text {for }}$ generated would increase with respect to the fraction of $\rho_{\text {rev }}$. In addition, the latter would vanish gradually due to annihilation. The present formulation, on the other hand, allows for increasing or decreasing the reversibility $P^{s}$ of a system, depending on whether the density of obstacles given by Eq. (11) increases or decreases. The latter is a loose representation of the current configuration of dislocation microstructures formed by dislocation-dislocation reactions. As shown in Fig. 9, the $P^{s}$ value in the representative slip system reduces at the beginning of deformation but presents a cyclic behavior at the saturation stage around a value not much lower than one.

The relative activity of cyclic shear loading with $\pm 30 \%$ of strain amplitude is presented in Fig. 10. It can be seen that about $70 \%$ of plastic deformation is contributed by $\{110\}\langle 111\rangle$ dislocations. Compared to the results of Kitayama et al. [25], the relative activities show a rapid transient at reloading due to the activation of the back-stress.

Following the Bauschinger effect, the work-hardening stagnation observed in the experimental results after each shear reversal is well predicted in the simulations. Such a behavior has been reported by many earlier works, i.e. $[9,16,34,48]$ and has been linked to the annihilation process of the re-mobilized dislocations upon strain reversal $[9,34]$, which is included in the present modeling framework. In this work the transient behavior is captured well due to the interplay between dislocation accumulation and annihilation during reloading. These two processes occur respectively on $\rho_{r e v}^{s+}$ and $\rho_{r e v}^{s-}$ at the same time but at different rates and strain interval, which is responsible for the low or even negative hardening rate in this stage. The parameter $m$ (Eqs. 5 and 7), which controls the 
annihilation rate, evidently affect the appearance of this behavior. Fig. 11 presents the evolution of reversible dislocation densities on a representative slip system of a single crystal tested in shear. It schematically shows that the plateau is more pronounced when the value of $m$ is reduced.

\section{Conclusions}

In this study, the crystallographic-based hardening model (RGBV model) is improved and extended with the intended aim to accurately predict the macroscopic stress-strain response for rolled low carbon steel in the context of strain path changes including cyclic loading and large plastic strains. The RGBV model, which is embedded in the VPSC framework, allows a non-monotonic evolution of the dislocation density during complex loadings paths through a suitable description of dislocation-related mechanisms.

A more sophisticated formulation of the dislocation reversibility parameter $P^{s}$ is proposed in this work. The new model calculates $P^{s}$ individually for each slip system, and allows $P^{s}$ to increase or decrease (instead of monotonically increase) according to the current status of the microstructure during dislocation accumulation and annihilation processes.

In comparison with the previous results [25], this version of the RGBV model achieves a promising improvement by reproducing the macroscopic hardening transient effects (plateau in the flow curve) during strain path changes, the Bauschinger effect (low yield stress following reload), strain hardening stagnation and large strain cyclic behavior (superimposed successive hysteresis loops). The new formulation of the reversibility parameter $P^{s}$ plays an important role to stabilize the mechanical response at very large 
cumulated strain.

Finally, by accounting explicitly for crystallography and the dislocation mechanisms that are responsible for hardening and shear accommodation, our model relies on a sound physical basis. While the constitutive equations proposed here are mostly empirical, they are qualitatively correct as far as the dependence on dislocation mechanisms. Most important, they can be improved with results obtained from basic Dislocation Theory models and lower scale Dislocation Dynamics simulations. Those are objectives of future work.

\section{Acknowledgements}

CT acknowledges support from US Department of Energy, Office of Basic Energy Science, Division of Materials Science and Engineering, Project FWP 06SCPE401DOEBES. WW, MB, GV, JG and FB acknowledge the financial support of FEDER funds through the Operational Program for Competitiveness Factors - COMPETE and National Funds through the FCT - Foundation for Science and Technology under the projects PTDC/EME-TME/105688/2008, PTDC/EME-PME/116683/2010, PESTC/EME/UI0481/2011, PTDC/EMS-TEC/0777/2012 and PEST-C/EME/UI0481/2013.

\section{References:}

[1] E. V. Nesterova, B. Bacroix, C. Teodosiu, Experimental observation of microstructure evolution under strain-path changes in low-carbon IF steel, Mater. Sci. Eng. A 309-310 (2001) 495-499.

[2] F. Barlat, J.M.F. Duarte, J.J. Gracio, A.B. Lopes, E.F. Rauch, Plastic flow for non- 
monotonic loading conditions of an aluminum sheet sample, Int. J. Plasticity 19 (2003) $1215-1244$.

[3] G. Vincze, E.F. Rauch, J.J. Gracio, F. Barlat, A.B. Lopes, A comparison of the mechanical behavior of an AA1050 and a low carbon steel deformed upon strain reversal, Acta Mater. 53 (2005) 1005-1013.

[4] E.F. Rauch, J.J. Gracio, F. Barlat, Work-hardening model for polycrystalline metals under strain reversal at large strain, Acta Mater. 55 (2007) 2939-2948.

[5] G. Proust, C.N. Tomé, A. Jain, S.R. Agnew, Modeling the effect of twinning and detwinning during strain-path changes of magnesium alloy AZ31, Int. J. Plast. 25 (2009) 861-880.

[6] J. Rossiter, A. Brahme, M.H. Simha, K. Inal, R. Mishra, A new crystal plasticity scheme for explicit time integration codes to simulate deformation in 3D microstructures: effects of strain path, strain rate and thermal softening on localized deformation in the aluminum alloy 5754 during simple shear, Int. J. Plasticity 26 (2010) 1702-1725.

[7] I.J. Beyerlein, C.N. Tomé, Modeling transients in the mechanical response of copper due to strain path changes, Int. J. Plasticity 23 (2007) 640-664.

[8] F. Barlat, J.J. Gracio, M.G. Lee, E.F. Rauch, G. Vincze, An alternative to kinematic hardening in classical plasticity, Int. J. Plasticity 27 (2011) 1309-1327.

[9] E.F. Rauch, J.J. Gracio, F. Barlat, G. Vincze, Modelling the plastic behavior of 
metals under complex loading conditions, Modell. Sim. Mater. Sci. Eng. 19 (2011) 1-18.

[10] M. Knezevic, R.J. McCabe, R.A. Lebensohn, C.N. Tomé, C. Liu, M.L. Lovato, B. Mihaila, Integration of self-consistent polycrystal plasticity with dislocation density based hardening laws within an implicit finite element framework: Application to lowsymmetry metals, J. Mech. Phys. Solids 61 (2013) 2034-2046

[11] J.-H. Schmitt, E.L. Shen, J.L. Raphanel, A parameter for measuring the magnitude of a change of strain path: validation and comparison with experiments on low carbon steel, Int. J. Plasticity 10 (1994) 535-551.

[12] D.V. Wilson, P.S. Bate, Reversibility in the work hardening of spheroidised steels, Acta Metall. 34 (1986) 1107-1120

[13] U.F. Kocks, Dislocation interactions: flow stress and strain hardening, 50th Anniversary Meeting on "Dislocations and Properties of Real Materials", London Institute of Metals, London (1985) 125-143

[14] P. Bate, D. Wilson, Analysis of the Bauschinger effect, Acta Metallica, 34 (1986) 1097-1105.

[15] N. Christodoulou, Evolution of the Bauschinger effect in tension and compression in Zircaloy-2, Acta Metall. 37 (1989) 529-539.

[16] M.G. Stout, A.D. Rollett, Large strain Bauschinger effects in FCC metals and alloys, Metall. Trans. 21A (1990) 3201-3213. 
[17] C.J. Bayley, W.A.M. Brekelmans, M.G.D. Geers, A comparison of dislocation induced back stress formulations in strain gradient crystal plasticity, Int. J. Solids Struct. 43 (2006) 7268-7286

[18] B. Peeters, B. Bacroix, C. Teodosiu, P. Van Houtte, E. Aernoudt, Workhardening/softening behaviour of b.c.c. polycrystals during changing strain:: Part II. TEM observations of dislocation sheets in an IF steel during two-stage strain paths and their representation in terms of dislocation densities. Acta Mater. 49 (2001) 1621-1632.

[19] P. Armstrong, C. Frederick, A Mathematical Representation of Multiaxial Bauschinger effect, CEGB Report rd/b/n731, Berkeley Nuclear Laboratories, Berkeley, UK, 1966.

[20] I.J. Beyerlein, C.N. Tomé, A dislocation based constitutive law for pure $\mathrm{Zr}$ including temperature effects, Int. J. Plasticity 24 (2008) 867-895.

[21] J.W. Yoon, F. Barlat, J.J. Gracio, E.F. Rauch, Anisotropic strain hardening behavior in simple shear for cube textured aluminum alloy sheets, Int. J. Plasticity 21 (2005) 2426-2447.

[22] G. Proust, C.N. Tomé, G.C. Kaschner, Modeling texture, twinning and hardening evolution during deformation of hexagonal materials, Acta Mater. 55 (2007) 2137-2148.

[23] M.G. Lee, H. Lim, B.L. Adams, J.P. Hirth, R.H. Wagoner, A dislocation densitybased single crystal constitutive equation, Int. J. Plasticity 26 (2010) 925-938. 
[24] D.W. Brown, I.J. Beyerlein, T.A. Sisneros, B. Clausen, C.N. Tomé, Role of twinning and slip during compressive deformation of beryllium as a function of strain rate, Int. J. Plasticity 29 (2012) 120-135.

[25] K. Kitayama, C.N. Tomé, E.F. Rauch, J.J. Gracio, F. Barlat, A crystallographic dislocation model for describing hardening of polycrystals during strain path changes. Application to low carbon steels, Int. J. Plasticity 46 (2013) 54-69.

[26] W. Wen, M. Borodachenkova, C.N. Tomé, G. Vincze, E.F. Rauch, F. Barlat, J.J. Grácio, Mechanical behavior of Mg subjected to strain path changes: Experiments and modeling, Int. J. Plasticity 73 (2015) 171-183.

[27] R.A. Lebensohn, C.N. Tomé, A self-consistent anisotropic approach for the simulation of plastic deformation and texture development of polycrystals-Application to zirconium alloys, Acta Metall. Mater. 41 (1993) 2611-2624.

[28] R.A. Lebensohn, C.N. Tomé, P. Ponte Castañeda, Self-consistent modeling of the mechanical behavior of viscoplastic polycrystals incorporating intragranular field fluctuations, Philos. Mag. 87 (2007) 4287-4322.

[29] N. Bertin, C.N. Tomé, I.J. Beyerlein, M.R. Barnett, L. Capolungo, On the strength of dislocation interactions and their effect on latent hardening in pure Magnesium, Int. J. Plasticity 62 (2014) 72-92.

[30] C.H. Cáceres, A.H. Blake, On the strain hardening behaviour of magnesium at room temperature, Mater. Sci. Eng. A 462 (2007) 193-196. 
[31] C.H. Cáceres, P. Lukáč, Strain hardening behaviour and the Taylor factor of pure magnesium, Philos. Mag. 88 (2008) 977-989.

[32] L. Capolungo, Dislocation junction formation and strength in magnesium, Acta Mater. 59 (2011) 2909-2917.

[33] L. Kubin, B. Devincre, T. Hoc, Modeling dislocation storage rates and mean free paths in face-centered cubic crystals, Acta Mater. 56 (2008) 6040-6049.

[34] B. Peeters, S.R. Kalidindi, P. Van Houtte, E. Aernoudt, A crystal plasticity based work hardening/softening model for BCC metals under changing strain paths, Acta Mater. 48 (2000) 2123-2133.

[35] B. Peeters, B. Bacroix, C. Teodosiu, P. Van Houtte, E. Aernoudt, Workhardening/softening behaviour of BCC polycrystals during changing strain paths: I. An integrated model based on substructure and texture evolution, and its prediction of the stress-strain behaviour of an IF steel during two-stage strain paths, Acta Mater. 49 (2001) $1607-1619$.

[36] S. Queyreau, G. Monnet, B. Devincre, Slip system interactions in a-iron determined by dislocation dynamic simulations, Int. J. Plasticity, 25 (2009) 361-377.

[37] U.F. Kocks, H. Mecking, Physics and phenomenology of strain hardening: the FCC case, Prog. Mater. Sci. 48 (2003) 171-273.

[38] U. Essmann, H. Mughrabi, Annihilation of dislocations during tensile and cyclic 
deformation and limits of dislocation densities, Phil. Mag. A 40 (1979) 731-756.

[39] H. Mughrabi, T. Ungar, W. Kienle, M. Wilkens, Long-range internal stresses and asymmetric X-ray line-broadening in tensile-deformed [001]-orientated copper single crystals, Phil. Mag. A 53 (1986) 793-813.

[40] X. Feaugas, On the origin of the tensile flow stress in the stainless steel AISI 316L at $300 \mathrm{~K}$ : back stress and effective stress, Acta Mater. 47 (1999) 3617-3632.

[41] H. Mughrabi, Dislocation wall and cell structures and long-range internal stresses in deformed metal crystals. Acta Metall. 31 (1983) 1367-1379.

[42] J.P. Hirth, J. Lothe, Theory of dislocations. Chapter 5. McGraw-Hill Book Comp, New York (1968).

[43] J.P. Hirth, On Dislocation Interactions in the fcc Lattice. J. Appl. Phys. 32 (1961) 700.

[44] M. Tang, L.P. Kubin, G.R. Canova, Dislocation mobility and the mechanical response of b.c.c. single crystals: A mesoscopic approach. Acta Mater. 46 (1998) 3221 3235 .

[45] R. Madec, L. Kubin, Second-order junctions and strain hardening in bec and fcc crystals. Scripta Mater.58 (2008) 767-770.

[46] H. Wang, B. Raeisinia, P.D. Wu, S.R. Agnew, C.N. Tomé, Evaluation of selfconsistent polycrystal plasticity models for magnesium alloy AZ31B sheet, Int. J. Solids 
Struct. 47 (2010) 2905-2917.

[47] E.F. Rauch, J.H. Schmitt, Dislocation substructures in mild steel deformed in simple shear, Mater. Sci. Eng. A113 (1989) 441-448.

[48] E.F. Rauch, Stress reversal tests imposed by shear on mild steel. In: Brandon, D.G. et al. (Eds.), Proceedings of ICSMA-9. Freund Publishing House, London, (1991) 187194. 

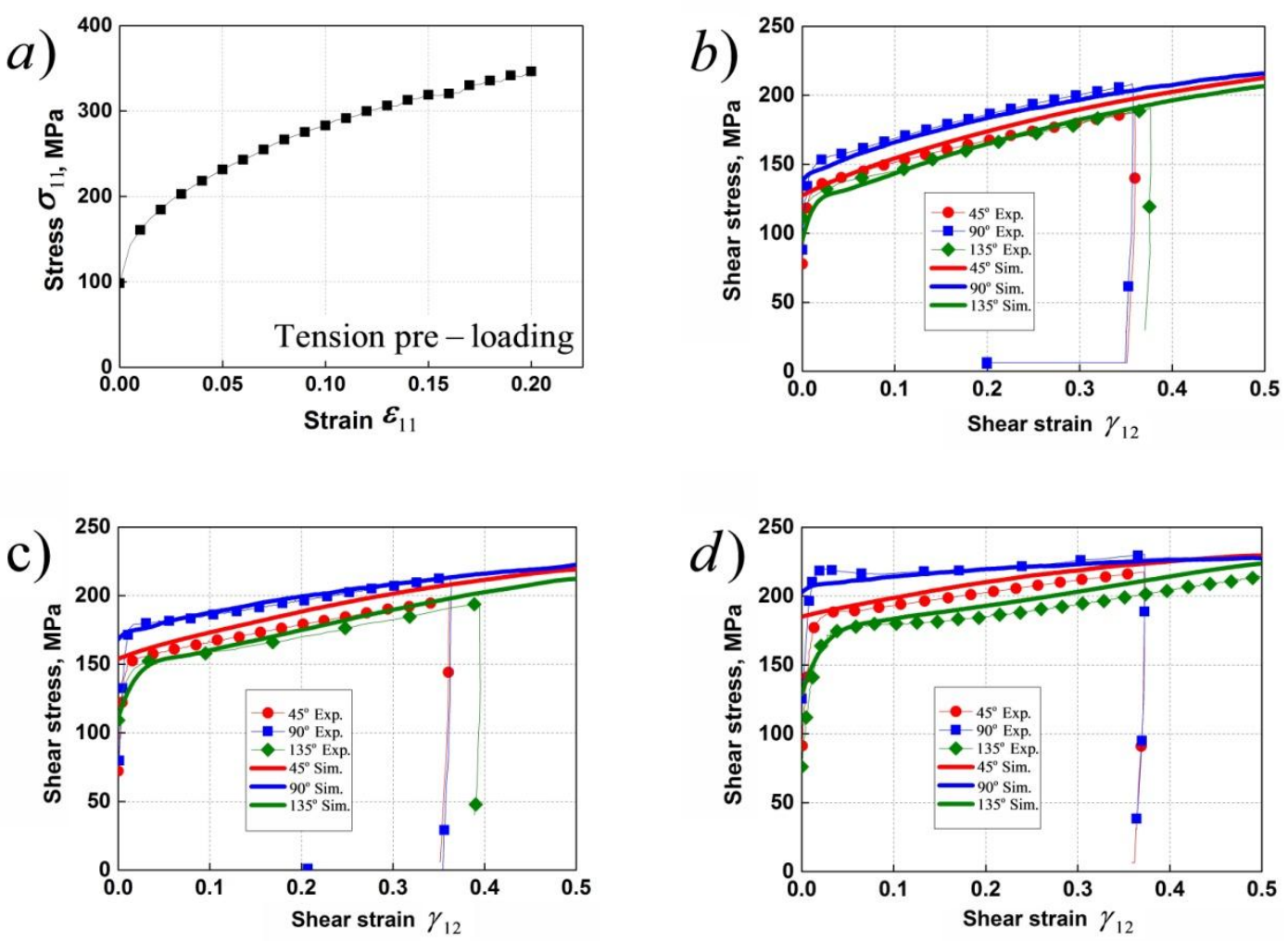

Fig. 1. (a) Predicted stress-strain response of rolled LC steel for tension along the RD;

(b) Predicted and measured shear stress-shear strain reloads at $45^{\circ}, 90^{\circ}, 135^{\circ}$ with respect to the $\mathrm{RD}$, after $5 \%$ tension preload; (c) idem after $10 \%$ preload; (d) idem after $20 \%$ preload. 
Figure 2

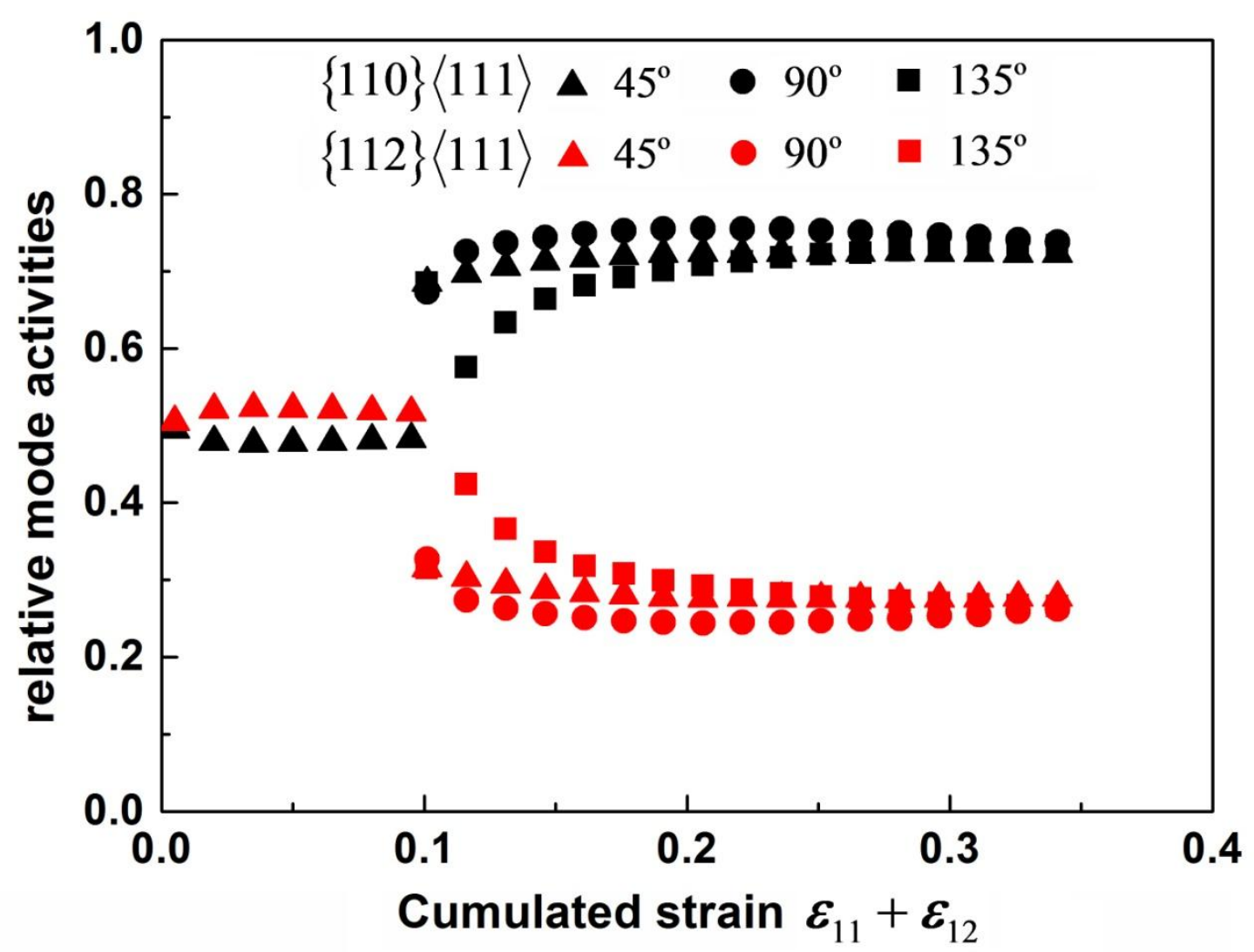

Fig. 2. Relative shear activity of $\{110\}\langle 111\rangle$ and $\{112\}\langle 111\rangle$ slip modes during $10 \%$ tension preload along RD followed by shear at $45^{\circ}, 90^{\circ}$ and $135^{\circ}$. 


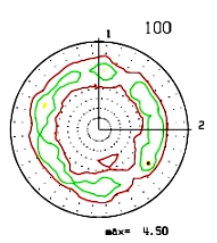

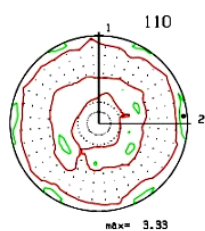

initial

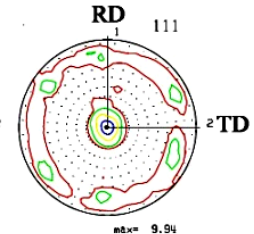

nexe= 9.94

(1)
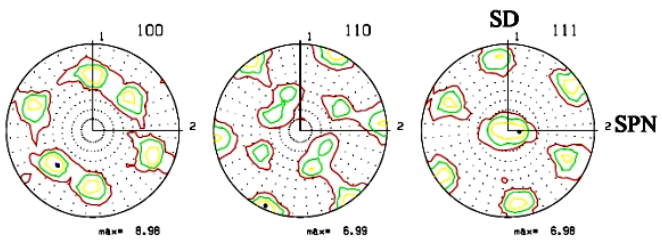

after $30 \%$ shear reloading at $90^{\circ}$
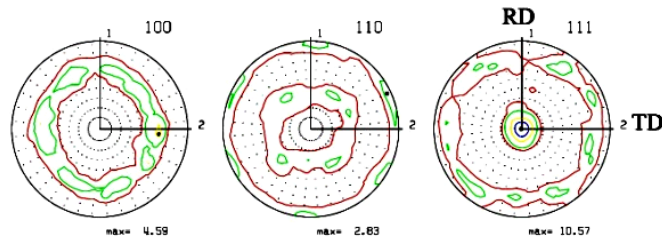

levels

1. 00

2.00

8. 00

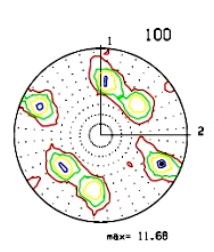

after $60 \%$ shear reloading at $90^{\circ}$

Fig. 3. Predicted texture evolution for tension-shear test with $10 \%$ tension pre-strain followed by simple shear reloading at $90^{\circ}$. SD is shear direction and SPN shear plane normal. 
Figure 4

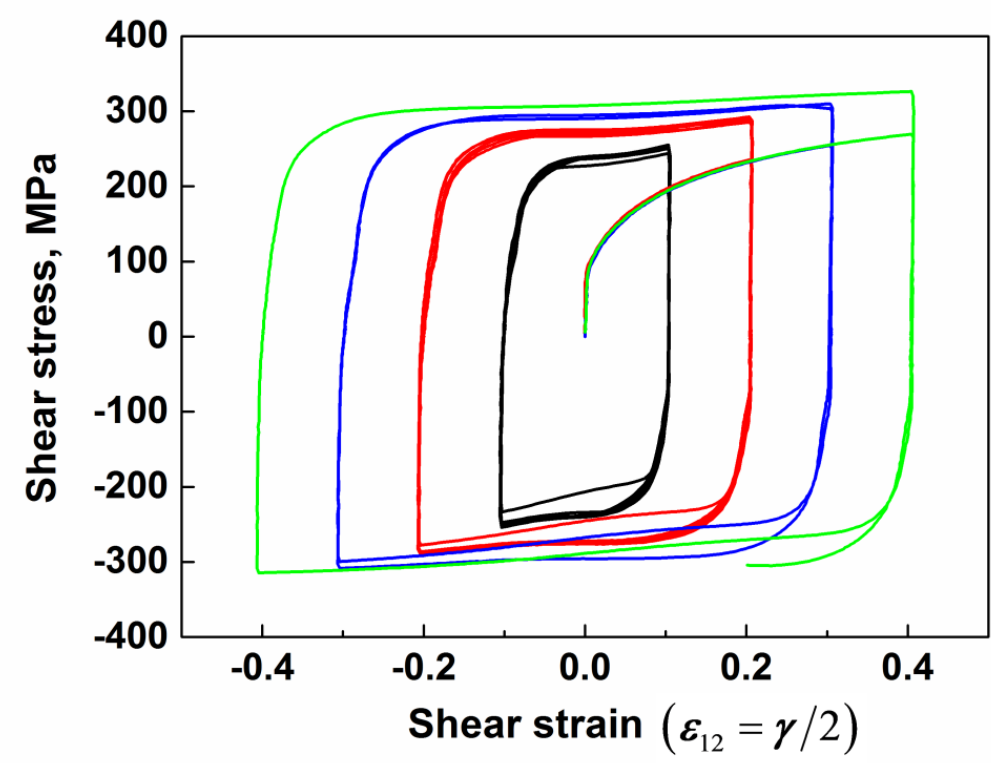

Fig. 4. Experimental response of LC steel subjected to reversed cyclic loading with maximum strain amplitudes of $\pm 10 \%$ ( 10 cycles), $\pm 20 \%$ ( 5 cycles), $\pm 30 \%$ ( 2 cycles) and $\pm 40 \%$ ( 1 cycle) 

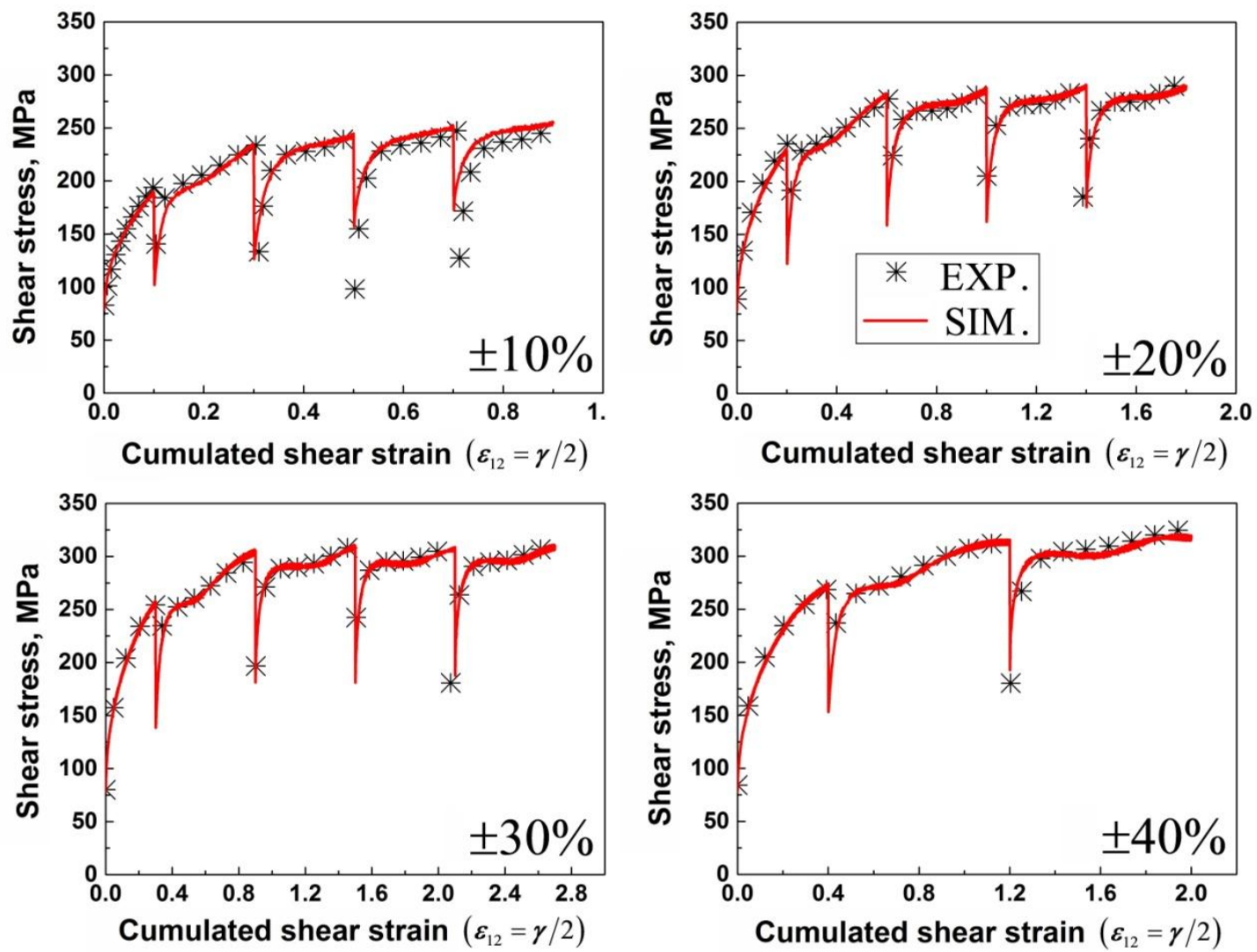

Fig. 5. Predicted stress-strain response for the cyclic tests of LC steel with different maximum strain amplitudes. The accumulated strain is used in the plot to facilitate comparison with experimental data 
Figure 6

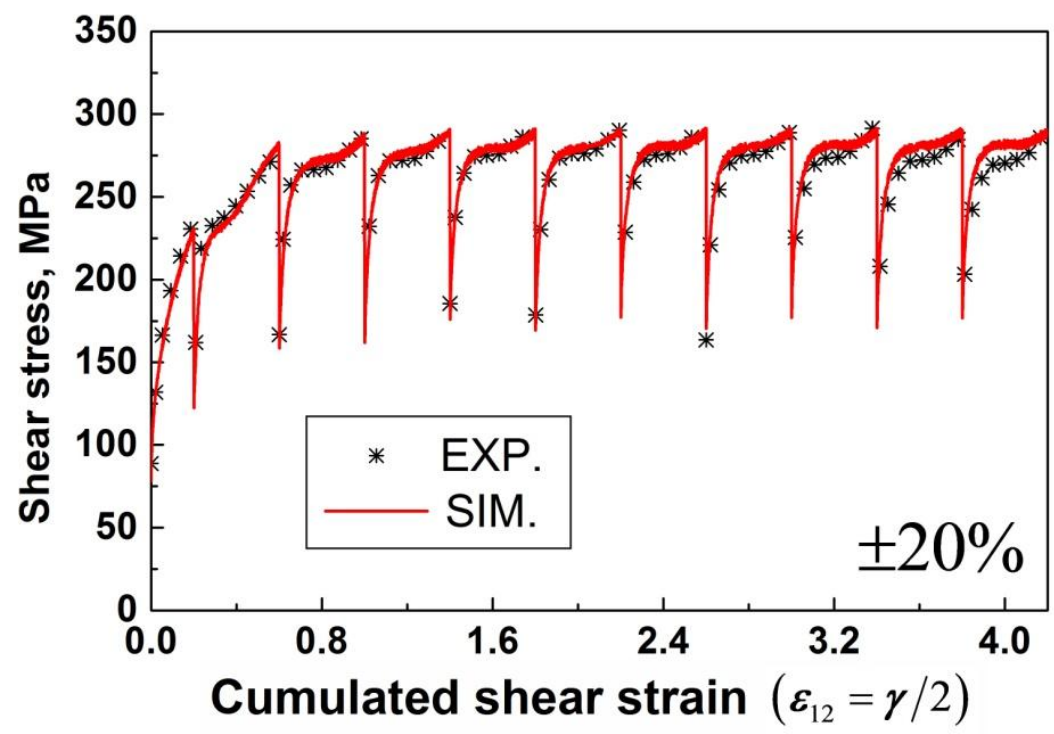

Fig. 6. Predicted stress-strain response of LC steel for 5 cycles shear-reverse shear test and strain amplitude of $\pm 20 \%$. The accumulated strain is used in the plot to facilitate comparison with experimental data. 


\section{Figure 7}
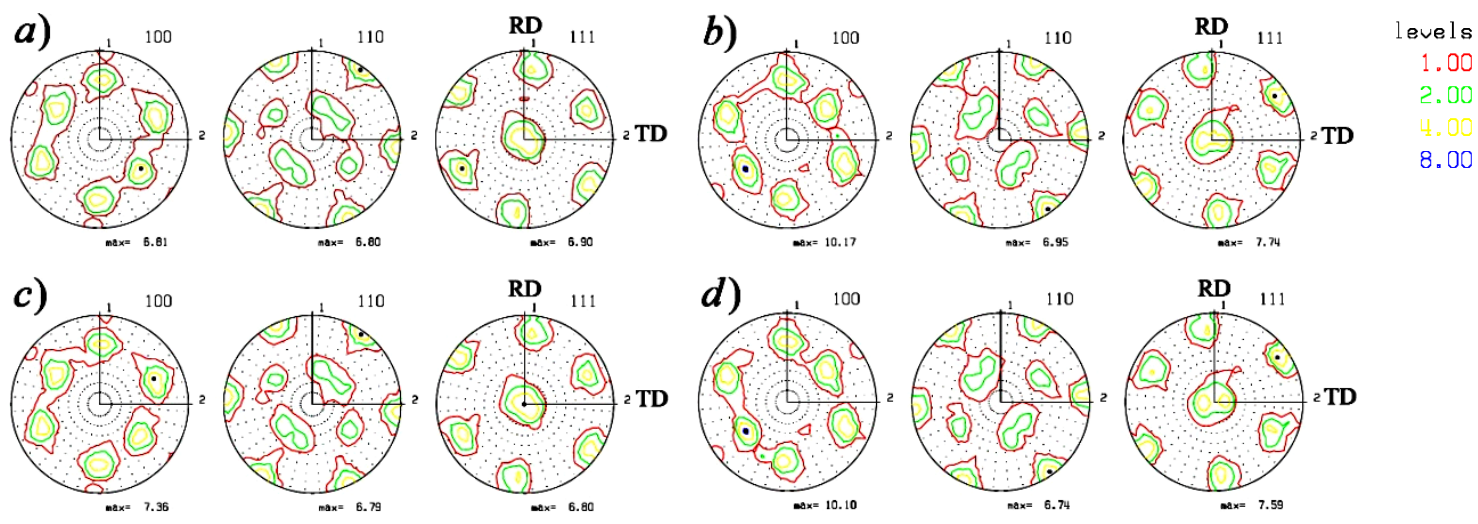

Fig. 7. Predicted texture evolution for cyclic shear test with $\pm 30 \%$ shear strain amplitude after 30\% (a), 90\% (b), 150\% (c) and 210\% (d) accumulated shear strain. 
Figure 8
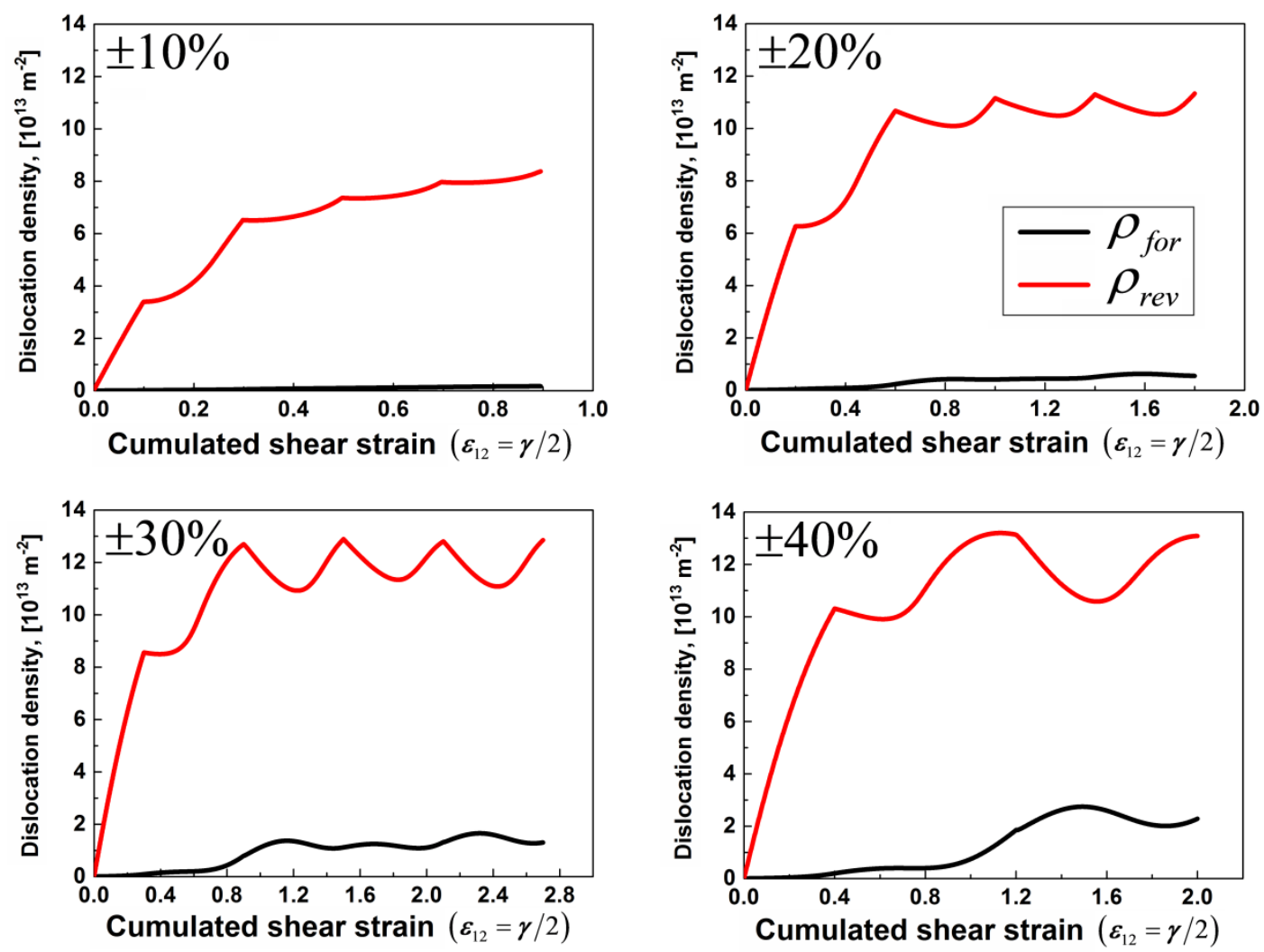

Fig. 8. Evolution of the total forward and reverse dislocation densities during cyclic loading in shear with different amplitudes 
Figure 9

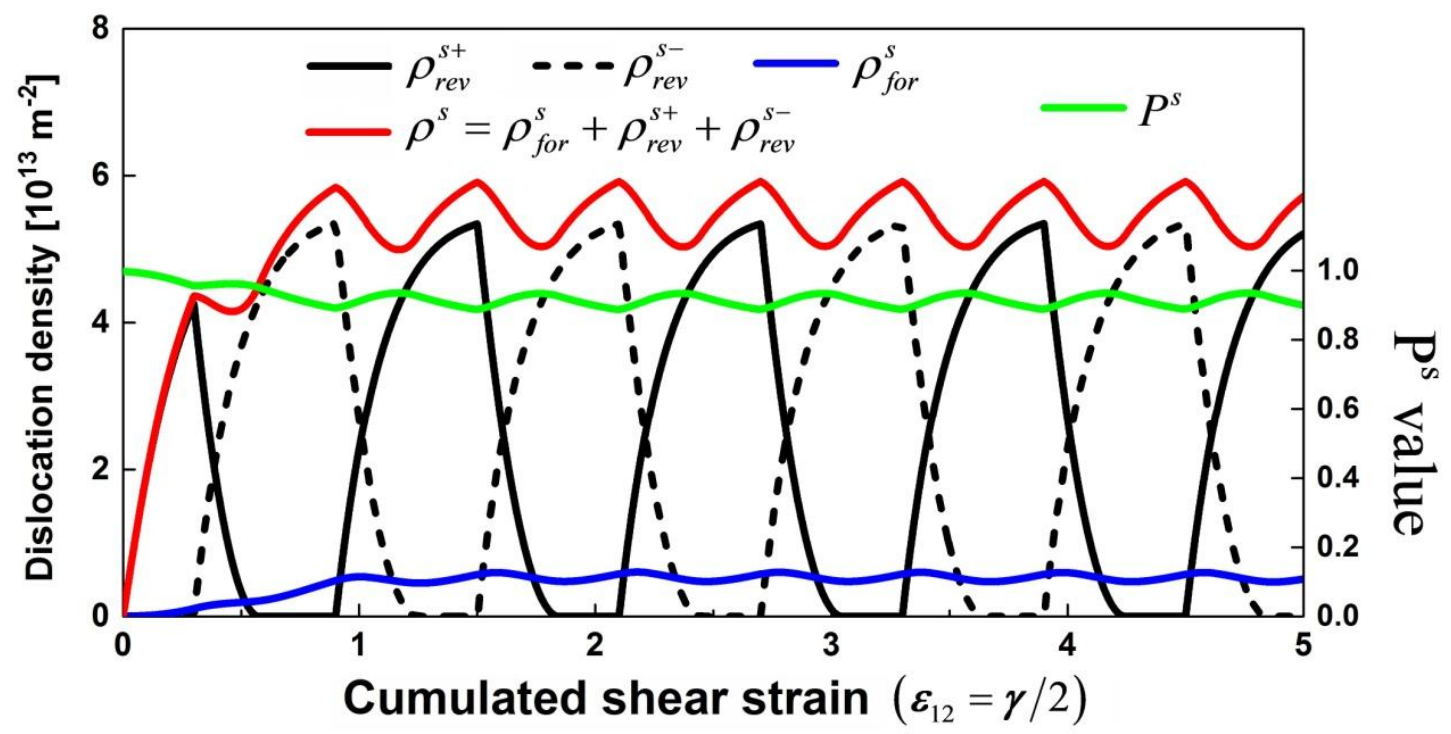

Fig. 9. Evolution of the forward and reversible dislocation density and $P^{s}$ value for one representative slip system during cyclic loading, obtained from a single crystal test. 
Figure 10

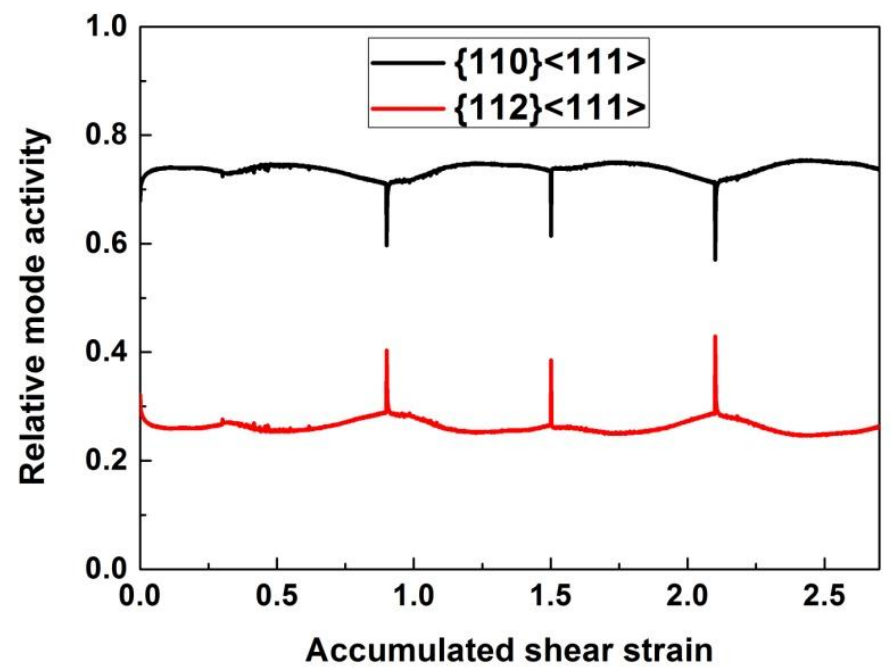

Fig. 10. Relative shear activities of $\{110\}\langle 111\rangle$ and $\{112\}\langle 111\rangle$ slip modes during cyclic shear loading with $\pm 30 \%$ shear strain amplitude. 
Figure 11

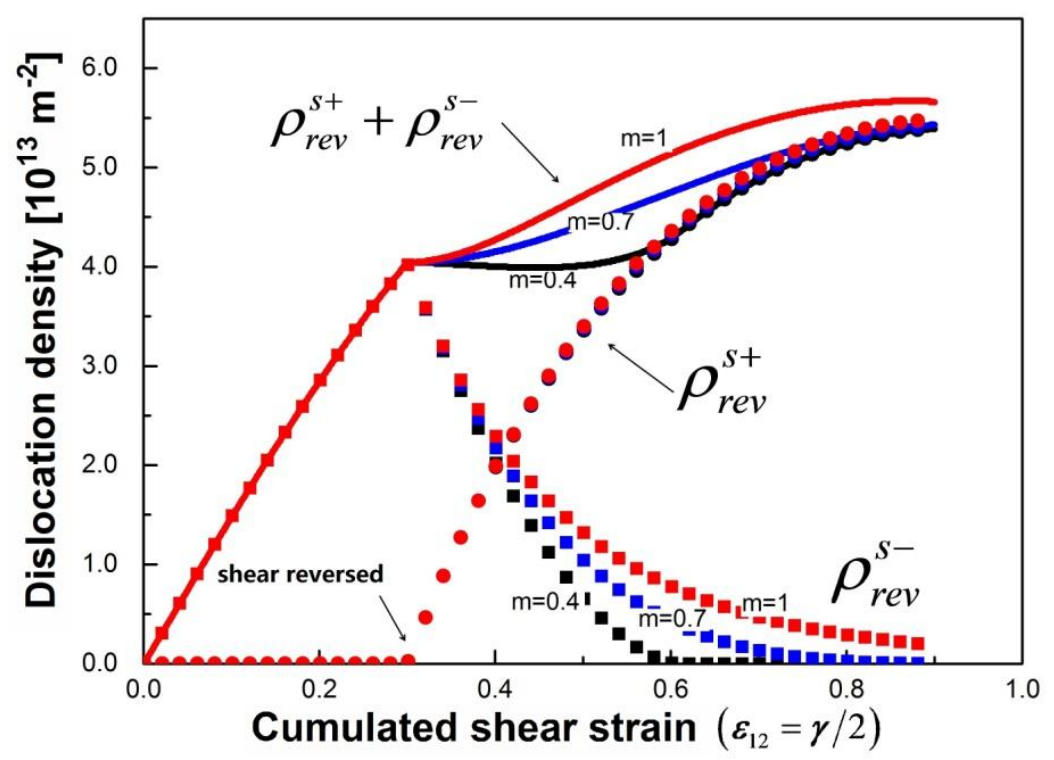

Fig. 11. Schematic evolution of $\rho_{r e v}^{s+}, \rho_{r e v}^{s-}$ and $\rho_{r e v}^{s+}+\rho_{r e v}^{s-}$ on one slip system when the shear is reversed 
Table 1. Hardening parameters for the LC steels used in this work

\begin{tabular}{ccc}
\hline Parameters & $\begin{array}{c}\text { Cyclic shear-shear } \\
\text { (steel \#1) }\end{array}$ & $\begin{array}{c}\text { Tension-shear } \\
\text { (steel \#2) }\end{array}$ \\
\hline$\mu$ (Elastic shear modulus) & $85 \mathrm{GPa}$ & $85 \mathrm{GPa}$ \\
\hline$b$ (Burgers vector) & $2.46 \cdot 10^{-10} \mathrm{~m}$ & $2.46 \cdot 10^{-10} \mathrm{~m}$ \\
\hline$D($ Grain size) & $30 \mu \mathrm{m}$ & $30 \mu \mathrm{m}$ \\
\hline$\tau_{o}$ (Initial CRSS) $(\{110\}<111>)$ & $55 \mathrm{MPa}$ & $38 \mathrm{MPa}$ \\
$\tau_{o}$ (Initial CRSS) $(\{110\}<111>)$ & $55 \mathrm{MPa}$ & $40 \mathrm{MPa}$ \\
\hline$K$ (Mobile to storage parameter) & 650 & 950 \\
\hline$f($ Recovery parameter) & 1.3 & 1.8 \\
\hline$\rho_{\min }$ (Lower reversibility threshold) & $10^{11} \mathrm{~m}^{-2}$ & $10^{11} \mathrm{~m}^{-2}$ \\
\hline$\rho_{\max }$ (Upper reversibility threshold $)$ & $10^{14} \mathrm{~m}^{-2}$ & 0.5 \\
\hline$f_{B}^{s}$ (back-stress parameter; Eq 8) & 0.5 & 10 \\
\hline$q$ (back-stress parameter; Eq 8) & 5 & 0.5 \\
\hline$m$ (recombination rate parameter; Eq 5) & 0.5 & $0.25\left(s=s^{\prime}\right)$ \\
\hline$\alpha^{s s^{\prime}}($ dislocation-dislocation interaction) & $0.25\left(s=s^{\prime}\right)$ & $0.52\left(s \neq s^{\prime}\right)$ \\
\hline
\end{tabular}




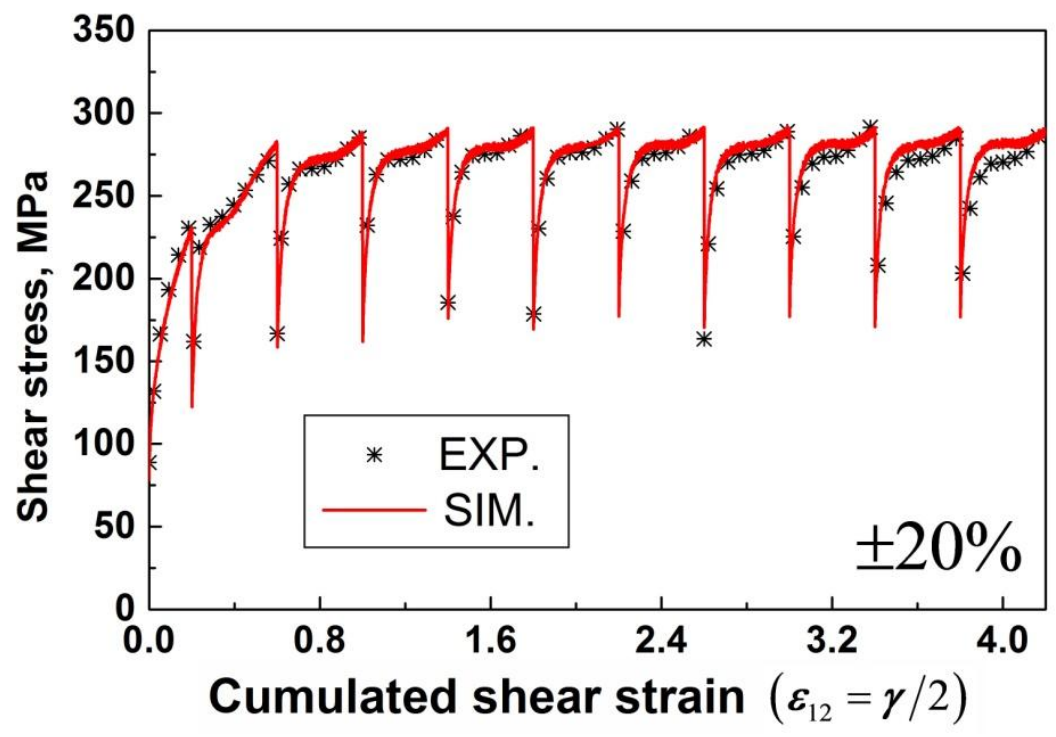

